\title{
MicroRNA-26a regulates glucose metabolism by direct targeting PDHX in colorectal cancer cells
}

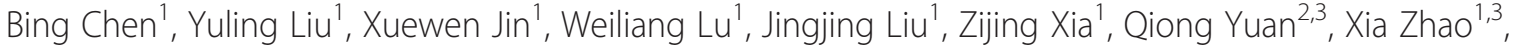 \\ Ningzhi $\mathrm{Xu}^{4}$ and Shufang Liang ${ }^{1 *}$
}

\begin{abstract}
Background: Reprogramming energy metabolism has been an emerging hallmark of cancer cells. MicroRNAs play important roles in glucose metabolism.

Methods: The targets of microRNA-26a (miR-26a) were predicted by bioinformatics tools. The efficacy of miR-26a binding the $3^{\prime}$-untranslated region (UTR) of pyruvate dehydrogenase protein X component (PDHX) mRNA was evaluated using a dual-luciferase reporter assay. The PDHX expression at the mRNA and protein level in several colon cancer cell lines was quantified with real-time PCR and Western blot analysis respectively. The effects of miR-26a on glucose metabolism were determined by detecting the content of glucose consumption, production of lactate, pyruvate, and acetyl-coenzyme A.

Results: The expression of miR-26a is inversely associated with the level of its targeting protein PDHX in several colon cancer cell lines with different malignancy potentials. MiR-26a inhibits PDHX expression by direct targeting the 3'-UTR of PDHX mRNA. The glucose consumption and lactate concentration were both greatly increased in colon cancer cells than the normal colon mucosal epithelia under physiological conditions. The overexpression of miR-26a in HCT116 cells efficiently improved the accumulation of pyruvate and decreased the production of acetyl coenzyme A. Meanwhile the inhibition of miR-26a expression induced inverse biological effects.
\end{abstract}

Conclusions: MiR-26a regulates glucose metabolism of colorectal cancer cells by direct targeting the PDHX, which inhibits the conversion of pyruvate to acetyl coenzyme A in the citric acid cycle.

Keywords: MicroRNA-26a, PDHX, Colorectal cancer, Glucose metabolism

\section{Background}

Recent studies have shown that microRNAs (miRNAs) play important roles in energy metabolism [1], and thus in cancer cells the alterations of cellular metabolism are associated with miRNA dysregulation [2,3]. MiRNAs participate in cell metabolism by regulating the expression of genes whose protein products either directly regulate metabolic machinery or indirectly modulate the expression of metabolic enzymes, serving as master regulators [4]. For example, microRNA-195-5p [5] and microRNA143 [6] can repress the glucose uptake and glycolysis processing by inhibiting the expression of glucose transporter 3 (GLUT3) and hexokinase 2 respectively. And

\footnotetext{
* Correspondence: zizi2006@scu.edu.cn

'State Key Laboratory of Biotherapy/Collaborative Innovation Center for Biotherapy, West China Hospital, Sichuan University, No.17, the third section of Renmin South Road, Chengdu 610041, P. R. China

Full list of author information is available at the end of the article
}

microRNA-143 could affect the glucose metabolism by regulating the AKT signaling pathway [7].

Reprogramming energy metabolism has been an emerging hallmark of cancer [8]. The best characterized metabolic phenotype in cancer cells is the "Warburg effect". Even in the presence of oxygen, cancer cells can reprogram their glucose metabolism, and thus the ATP generation shifts from oxidative phosphorylation to glycolysis, leading to a state termed "aerobic glycolysis" [9]. Unlike normal cells, cancer cells usually derive a substantial amount of energy from aerobic glycolysis by converting most incoming glucose to lactate rather than metabolizing it through oxidative phosphorylation. Although ATP production via glycolysis can be more rapid than by oxidative phosphorylation, the ATP generated is far less for per unit of glucose consumed. As a result, this shift demands that cancer cells implement an 
abnormally high rate of glucose uptake to meet their increased energy and biosynthesis. By now, several studies have focused on miRNA contributions to the "Warburg effect" $[1,3]$, and many molecules regulated by miRNAs are investigated.

MicroRNA-26a (miR-26a) shows a higher expression level in colon cancer tissues than in normal colon tissues [10], and its expression is also elevated under hypoxia conditions [11]. Meanwhile, miR-26a has been reported to play functions in cellular differentiation, cell growth, cell apoptosis and metastasis [12-15]. However, none of these studies on miRNA-26a focuses on energy metabolism. Several glycolysis genes, including glyceraldehyde3-phosphate dehydrogenase, phosphoglycerate kinase 1 and phosphoglycerate mutase 1 , have up-regulation in colon cancer tissues [16], therefore colon cancer cells have a higher glycolytic rate compared with normal cells [17]. In this study, we aim to discover the function of miR-26a on glucose metabolism in colorectal cancer cells. We have identified the pyruvate dehydrogenase protein X component (PDHX) as a direct target of miR26a that is involved in the biological processes of glucose metabolism in colorectal cancer (CRC) cells.

\section{Methods}

\section{Cell culture}

The CRC cell lines including SW620, SW480 and HCT116 were ordered from American Type Culture Collection. The cell line NCM460, which is derived from normal human colon mucosal epithelium, was ordered from the INCELL Corporation, LLC, USA (www.incell.com). The cells were all cultured in Dulbecco's modified Eagle's medium (DMEM) supplemented with 10\% fetal bovine serum at $37^{\circ} \mathrm{C}$ in humidified atmosphere with $5 \%(\mathrm{v} / \mathrm{v}) \mathrm{CO}_{2}$.

\section{Prediction of candidate miRNA targets}

The alignment of the miR-26a seed region and 3'-untranslated regions (UTR) of PDHX mRNA of different species were analyzed as literatures described previously $[6,18]$. The possible targets of miR-26a were screened by bioinformatics tools, Pictar (http://pictar.mdc-berlin.de) and TargetScan (http://www.targetscan.org).

\section{Construction of expression plasmids}

A 461-bp nucleotide fragments in pre-miR-26a sequences (Additional file 1: Table S1) were cloned into the pENTRCMV-EGFP vector (ordered from Wuhan Cell marker Biotechnology Co., Ltd., Wuhan, P. R. China) between $X h o I$ and EcoRI to construct miR-26a expression plasmid, pENTR-miR-26a. The empty vector pENTR-MIRNA was used as a control in the ectopic overexpression of miR-26a.

The 3'-untranslated region (3'UTR) of PDHX mRNA (Additional file 2: Table S2) was amplified by RT-PCR. The cDNA fragment corresponding to the 3'UTR of
PDHX mRNA was cloned in the downstream of the Renilla luciferase gene in the psiCHECK-2 vector (Cat. \# C8021, Promega, USA), which contains a reporter gene Renilla luciferase and an intraplasmid transfection normalization gene, a firefly luciferase. The 3'UTR of PDHX mRNA contains eight nucleotides $\left(5^{\prime}\right.$...UACUUGAA.... $\left.3^{\prime}\right)$, which are corresponding to miR-26a seed sequences $\left(3^{\prime}\right.$... AUGAACUU 5') (Figure 1A(I)). In the wild type recombinant plasmid pwt-PDHX, the relevant eight nucleotides (...TACTTGAA...) were involved (Figure 1A(II)). Meanwhile, in the mutant recombinant plasmid pmt-PDHX (Figure $1 \mathrm{~A}(\mathrm{III})$ ), the eight nucleotides were mutated into a random nucleotide sequence (...TCACCAAT...).

\section{Cells treated with MiR-26a inhibitor}

The miR-26a inhibitor was commercially available chemically modified antisense oligonucleotide inhibitor (Product ID: MH10249, Cat. \#4464084, Life Technologies Corporation, USA). The non-targeting oligonucleotides

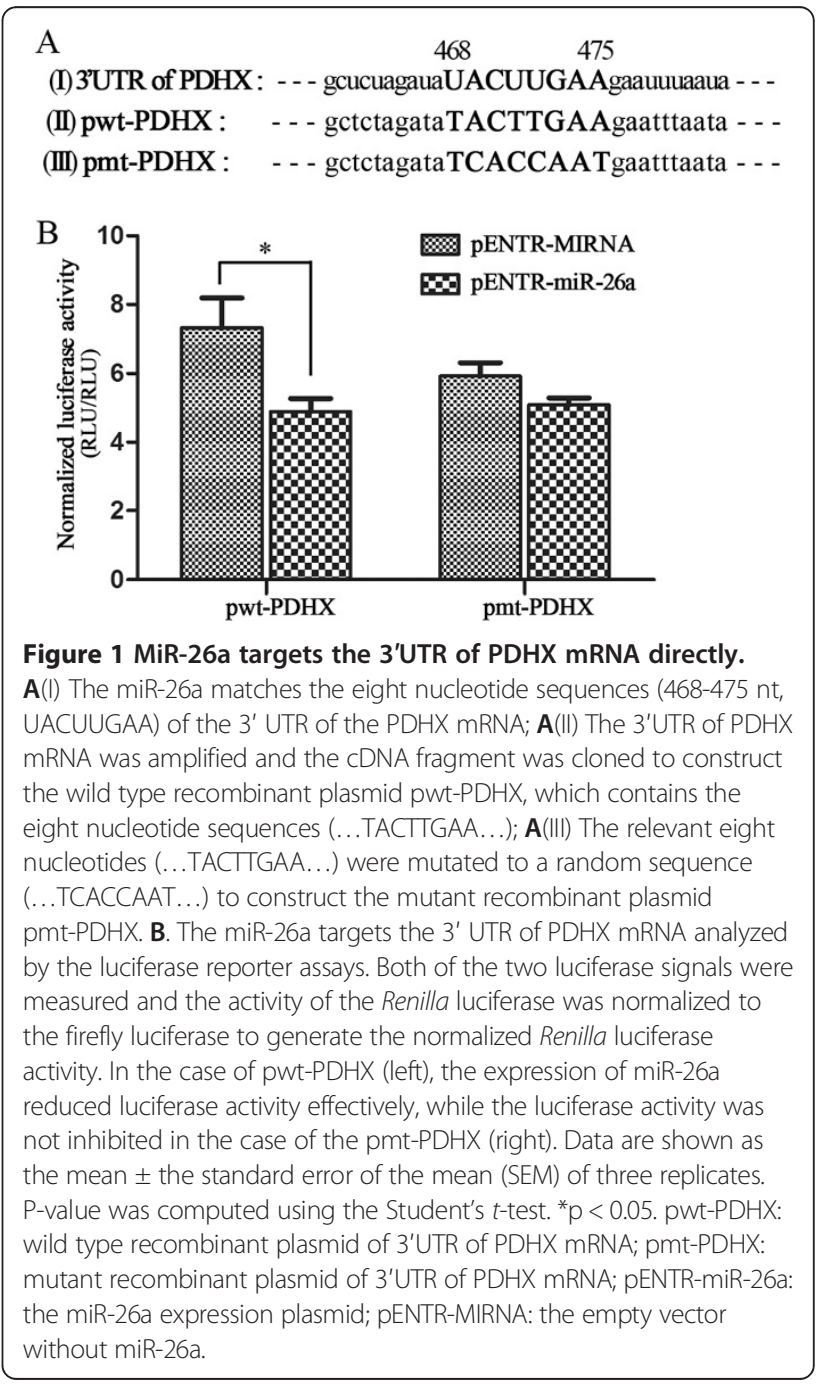


(Cat. \#4464076, Life Technologies Corporation, USA), as a negative control, were also ordered from this company.

The miR-26a inhibitor or the negative control was respectively transfected at a working concentration of 30 nM using Lipofectamine 2000 reagent (Cat. \#11668-019, Invitrogen). The ratio of Lipofectamine 2000 reagent $(\mu \mathrm{L})$ versus the oligonucleotides (pmol) was 18:1.

\section{RNA extraction and real-time PCR}

Total RNA was extracted using Trizol reagent (Cat. \#15596026, Invitrogen). The total RNA was dissolved with RNasefree water for use. The first-strand cDNA for miR-26a was generated using a cDNA synthesis kit (Cat. \#K1622, Thermo Scientific). Specific primers were designed for the reverse transcription of miR-26a and U6. The primer sequences for miR-26a were TGTCAACGATACGCTACCTAACGGCATGACAGTGTCAGCCTA. And the primer for U6 was GAACGCTTCACGAATTTGC based on literature reports [13]. For PDHX, random primers were used for mRNA reverse transcription (Cat. \#170-8890, Bio-Rad).

The real-time PCR was performed to measure the relative expression using the Supermix-Bio-Rad kit (Cat. \#172-5261, Bio-Rad) following the protocol. The U6 was used as an internal reference to calculate the relative expression level of miRNA, and the GAPDH mRNA level was taken as a comparison base for the target RNA expression. The relative RNA expression was calculated with the comparative CT method, which was normalized to the internal references. The forward RT-PCR primer for PDHX was 5'-GTC CCTCTAAAGCAGCTCAAAA-3', and the reverse one was 5'-CTCCCTTCAAAAGATCCAACTG-3'. The forward one for miR-26a was $5^{\prime}$-CTGTCAACGATACGCT AC-3', and its reverse was 5'-GTAATCCAGGATAG GCTG-3'. In addition, the forward RT-PCR primer for U6 was 5'-CTTCGGCAGCACATATAC-3', meanwhile its reverse primer was $5^{\prime}$-GAACGCTTCACGAATTTGC-3'.

\section{Cell transfection}

The miR-26a expression plasmid pENTR-miR-26a or the empty vector pENTR-MIRNA was respectively transfected at a working concentration of $100 \mathrm{nM}$ using Lipofectamine 2000 reagent (Cat. \#11668-019, Invitrogen). The ratio of DNA $(\mu \mathrm{g})$ versus the Lipofectamine 2000 reagent $(\mu \mathrm{L})$ was $1: 2$.

For the promoter luciferase reporter assay, the plasmid pwt-PDHX or pmt-PDHX was co-transfected with the miR-26a expression vector pENTR-miR-26a or the empty vector pENTR-MIRNA into HEK293T cells. As mentioned above, Lipofectamine 2000 was used as the transfection reagent. The plasmid was used at a working concentration of $100 \mathrm{nM}$ as well. The ratio of plasmid DNA $(\mu \mathrm{g})$ versus the Lipofectamine 2000 reagent $(\mu \mathrm{L})$ was 1: 2.

\section{Western blot analysis}

The protein PDHX was detected against its specific antibody by western blot. The PVDF membranes were respectively incubated with the primary antibody of PDHX (diluted 1:1000, Cat. \# S0394, Epitomics, USA) at $4^{\circ} \mathrm{C}$ overnight, followed by incubating with a secondary HRP-conjugated antibody at $37^{\circ} \mathrm{C}$ for $1 \mathrm{~h}$. Signal detection was performed with Luminata Crescendo Western HRP Substrate (Cat. \# WBLUR0100, Millipore). The detection of GAPDH using its antibody (Cat. \# sc-365062, Santa Cruz Biotechnology) was taken as a control.

\section{Luciferase reporter assay}

Either the plasmid pwt-PDHX or pmt-PDHX was respectively co-transfected with the miR-26a expression vector pENTR-miR-26a into HEK293T cells, and cells were harvested for assessment of luciferase activity at 48 hours after transfection. The luciferase activities of cellular extracts were measured with a Dual Luciferase Reporter Assay System (Cat. \#E1910, Promega). Both Renilla luciferase and firefly luciferase activities were measured. The luciferase signal was normalized to the firefly luciferase signal as described previously [19].

\section{Measurement of glucose consumption and lactate production}

Either the pENTR-miR-26a or miR-26a inhibitor was transfected into CRC cells. Cell culture media were collected after transfection for $48 \mathrm{~h}$. Glucose uptake and lactate production were measured using Amplex ${ }^{\oplus}$ Red Glucose/Glucose Oxidase Assay Kit (Cat. \#A22189; Invitrogen) and lactate assay kit (Cat. \#MAK064; SigmaAldrich) respectively. The results were normalized on the basis of total cellular protein amounts.

\section{Pyruvate assay}

The concentration of pyruvate in CRC cells, transfected with pENTR-miR-26a or miR-26a inhibitor, was respectively measured using pyruvate assay kit (Cat. \#K609-100; BioVision). Briefly, cells were collected after transfection for $48 \mathrm{~h}$ and dissolved with $0.5 \mathrm{ml}$ of pyruvate assay buffer. And $50 \mu \mathrm{l}$ sample was added with $50 \mu \mathrm{l}$ of reaction mixture to incubate at room temperature for 30 minutes. A standard curve covering a range of $10-0.1 \mathrm{nmol}$ per well was used as control. Absorbance was measured at $570 \mathrm{~nm}$. The pyruvate concentration, which was normalized on the basis of totally cellular protein amounts, was calculated relative to the standard curve.

\section{Acetyl-coenzyme aassay}

To analyze the production of acetyl coenzyme A (acetylCoA), cell extracts were prepared using the perchloric acid approach as described previously, with minor modification [20]. CRC cells, treated with pENTR-miR-26a or 
miR-26a inhibitor, were harvested and washed with phosphate-buffered saline. Cells were mixed in $1 \mathrm{~mL}$ of washing buffer (10 $\mathrm{mM}$ sodium phosphate [pH 7.5], $10 \mathrm{mM} \mathrm{MgCl} 2,1 \mathrm{mM}$ EDTA), treated with $200 \mu \mathrm{L}$ of $3 \mathrm{M}$ ice-cold $\mathrm{HClO}_{4}$, and incubated on ice for $30 \mathrm{mi}$ nutes. The mixture was centrifuged for 5 minutes at $10,000 \times \mathrm{g}$ at $4^{\circ} \mathrm{C}$. The supernatant was neutralized with saturated $\mathrm{KHCO}_{3}$ and centrifuged as described above. The level of acetyl-CoA in the cell extraction was quantified using the acetyl-CoA assay kit (Cat. \# K317-100; BioVision). Fluorescence was measured $(\mathrm{Ex} / \mathrm{Em}=535 /$ $589 \mathrm{~nm})$, and the acetyl-CoA concentration was calculated based on the standard curve. The production of acetyl-CoA was normalized based on the total of cellular proteins.

\section{Results}

\section{Bioinformatics analysis for potential targets of miR-26a}

By analyzing the 3'UTR mRNA of several glycolytic enzymes, we found that the 3'UTR of PDHX is relatively long with around 797 nucleotides, suggesting that PDHX mRNA is a possible target for miRNAs. In order to predict the possible target gene of miR-26a, the miRNA binding sites in the 3'UTR of PDHX were further analyzed through the Pictar and TargetScan software $[6,18]$. A conserved miR-26a binding site exists in the 3'UTR of PDHX across Homo sapiens (Hsa), Pan troglodytes (Ptr) and Mus musculus (Mmu) (Figure 2A), further we predicted an exact match to the seed region of mature miR26a (Figure 2B). The bioinformatics analysis indicated a potential functional link between PDHX and miR-26a.

\section{MiR-26a level is inversely associated with the protein expression of PDHX}

The expression level of miR-26a in NCM460, HCT116, SW480 and SW620 cells was examined using q-PCR analysis (Figure 2C). Compared with the normal epithelial cells NCM460, the expression of miR-26a was significantly increased to 1.3, 1.7 and 4.6-fold for the colon cancer cell lines HCT116, SW480 and SW620 respectively $(\mathrm{p}<0.05)$. Generally, the expression level of miR26a was significantly higher in malignant CRC cell lines HCT116, SW480 and SW620 than in a normal colorectal mucosal epithelial cell line NCM460 [21], especially it was almost increased over 4-fold in SW620 cells, which has high lymph node metastatic potentials [22]. Among the three CRC cell lines, the expression levels of miR-26a were gradually increased from HCT116 to SW480 and SW620 cells. Compared with HCT116 cells, which is derived from a human colorectal adenocarcinoma [22], SW480 and SW620 cells are typical model systems for CRC metastasis. The SW480 is derived from the primary site of CRC and SW620 comes from the recurrent lymph node metastasis [23]. Therefore, it has

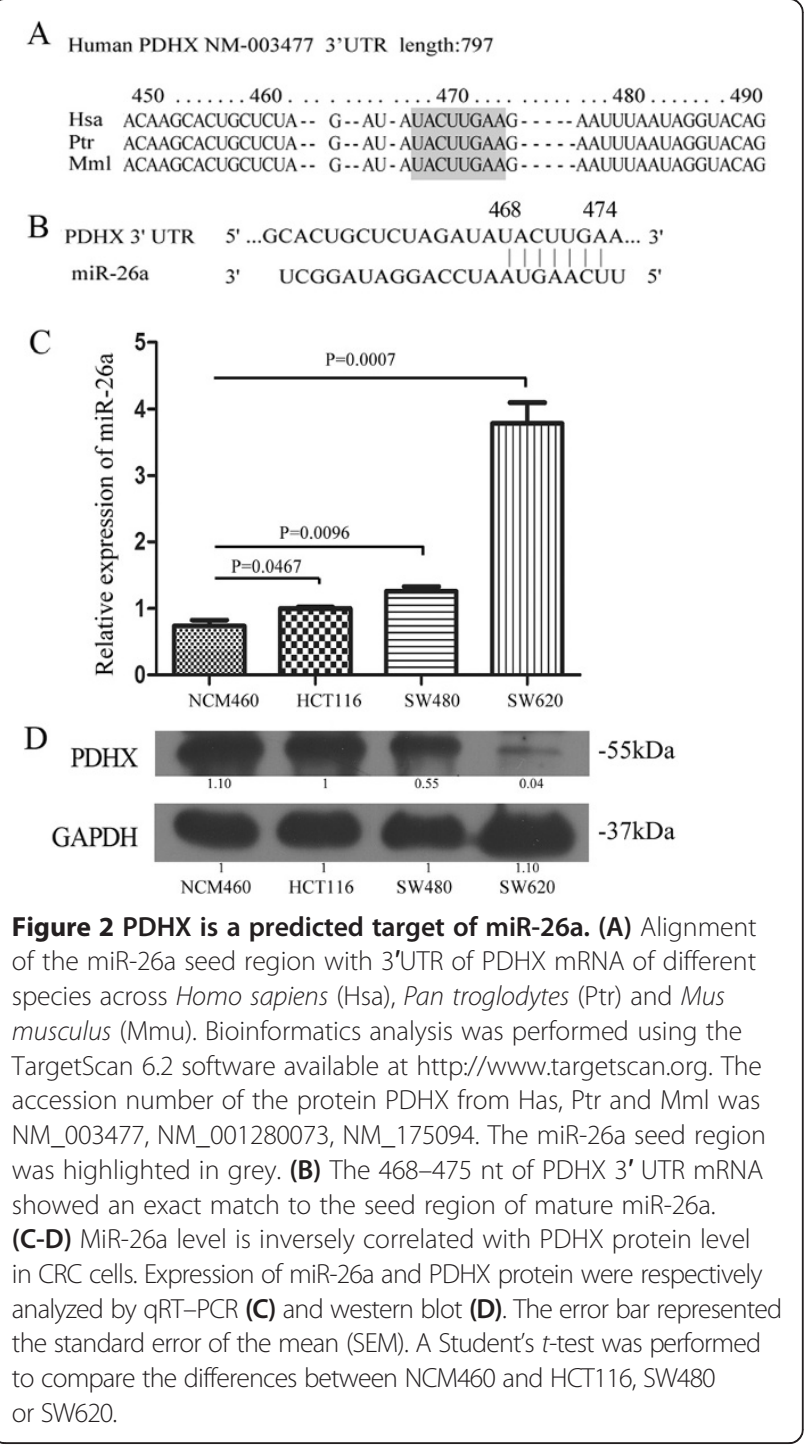

a positive correlation between the expression levels of miR-26a and the malignant degree of CRC cells.

As miRNA expression is often inversely correlated with those of their specific target mRNAs, we further analyzed the protein expression of the miR-26a target PDHX, in CRC cell lines. We found that the expression of PDHX was stepwise decreased in NCM460, HCT116, SW480 and SW620 cells (Figure 2D). The expression level of miR-26a in NCM460 was the lowest among the four cell lines (Figure 2C), but the protein level of PDHX exhibited the highest (Figure 2D). While in SW620 cells, the highest level of miR-26a expression and the lowest level of PDHX expression were observed. By comparing the expression levels of miR-26a and PDHX, an inverse correlation between them was observed in CRC cell lines, which supported the bioinformatics prediction on miR-26a target as above. 


\section{PDHX is a direct target of miR-26a}

Among these CRC cell lines, HCT116 cells were chosen to perform the following studies since it showed the media expression of miR-26a. Firstly, the miR-26a expression plasmid pENTR-miR-26a was transfected into HCT116 cells to detect its influence on the expression level of PDHX. As a result, a marked reduction of PDHX was observed at both mRNA and protein level when miR-26a was overexpressed with almost 2.5-fold (Figure 3A). Compared to the empty vector pENTRMIRNA, the expression of PDHX mRNA reduced almost $33 \%(\mathrm{P}<0.001)$ in HCT116 cells transfected with pENTR-miR-26a (Figure 3B), and PDHX protein was decreased by almost 40\% (Figure 3C).

Furthermore, the suppression of miR-26a expression was performed in HCT116 cells treated with $30 \mathrm{nM}$ of miR-26a inhibitor, and the random oligonucleotides were taken as negative controls. Compared with the negative control, the mRNA expression of PDHX had a 1.3-fold upregulation (Figure 3E) in HCT116 cells, along with about $70 \%$ inhibition of miR-26a (Figure 3D), and the protein expression of PDHX was increased 1.5-fold in the treated HCT116 cells (Figure 3F).

We also performed the loss-of function studies in SW620 cells, which have a highly endogenous expression level of miR-26a and a low level of PDHX (Figure 2C-D). Under the condition of a great down-regulation of miR$26 \mathrm{a}$ in SW620 cells (Figure 4A, p $<0.01$ ), the mRNA expression of PDHX was significantly increased with 2-fold (Figure 4B, p < 0.01), while the protein level of PDHX was improved to 1.56 -fold (Figure 4C). The down-regulation of endogenous miR-26a in SW620 cells could restore PHDX expression, which is similar to the effects with a knock-down of miR-26a in HCT116 cells (Figure 3).

Except to the gain and loss-of function studies, the direct regulatory effect of miR-26a on PDHX expression was also verified by Renilla luciferase reporter gene assays. Either the plasmid pwt-PDHX or pmt-PDHX was co-transfected with the miR-26a-expressing pENTRmiR-26a into HEK293T cells respectively. Cells were harvested for assessment of luciferase activity at 48 hours after transfection. Compared with the mutated reporter

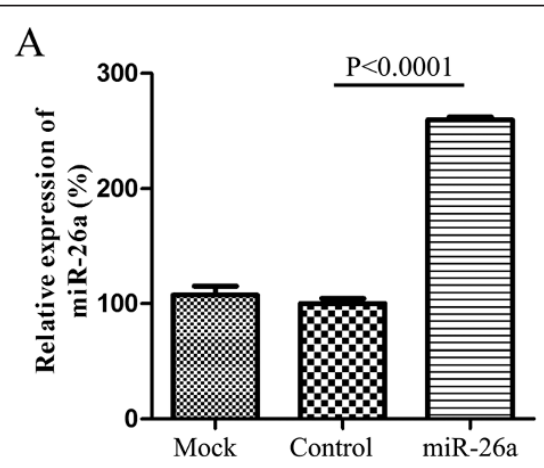

$\mathrm{B}$

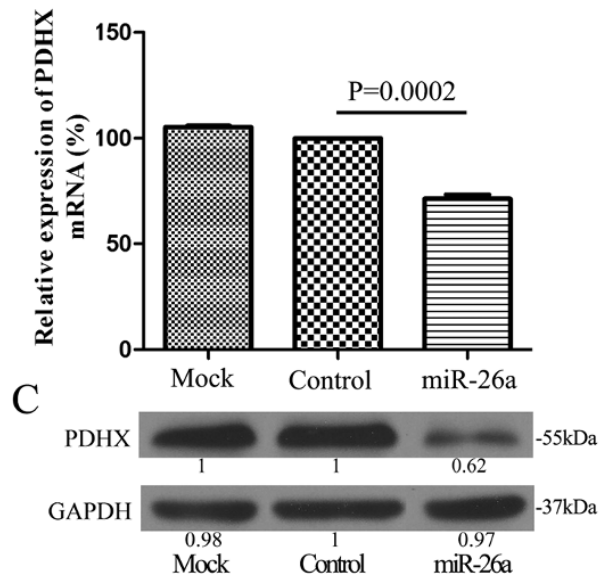

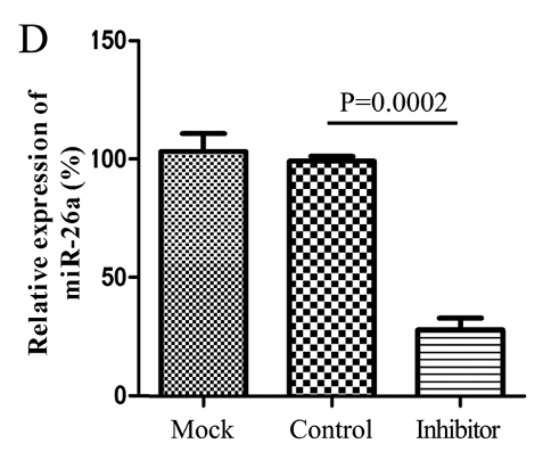

$\mathrm{E}$
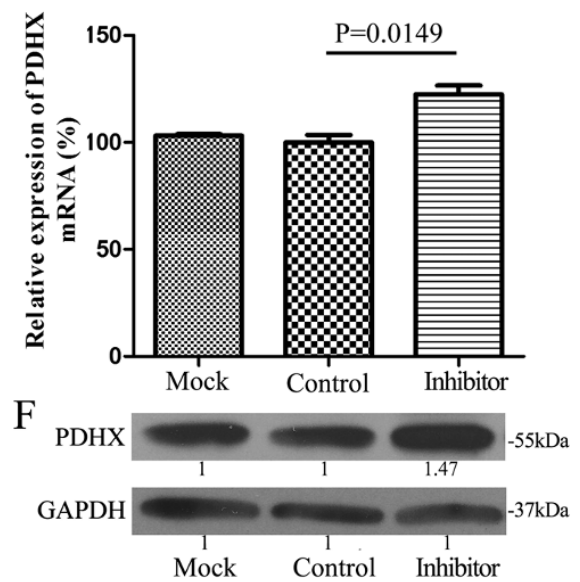

Figure 3 A negative correlation between the expression level of miR-26a and PDHX in HCT116 cells. The ectopic overexpression of miR-26a (A) induced a significant reduction of PDHX expression both in mRNA (B) and protein levels (C) in HCT116 cells. The empty plasmid pENTR-MIRNA was used as a control. While the expression of PDHX at both mRNA and protein levels $(\mathbf{E}, \mathbf{F})$ was increased in HCT116 cells along with an inhibition of miR-26a expression (D). The non-targeting oligonucleotide was used as a control. The untreated HCT116 cells were taken as the mock object. The mean values were calculated from three separate experiments. SEM: the standard error of the mean. 


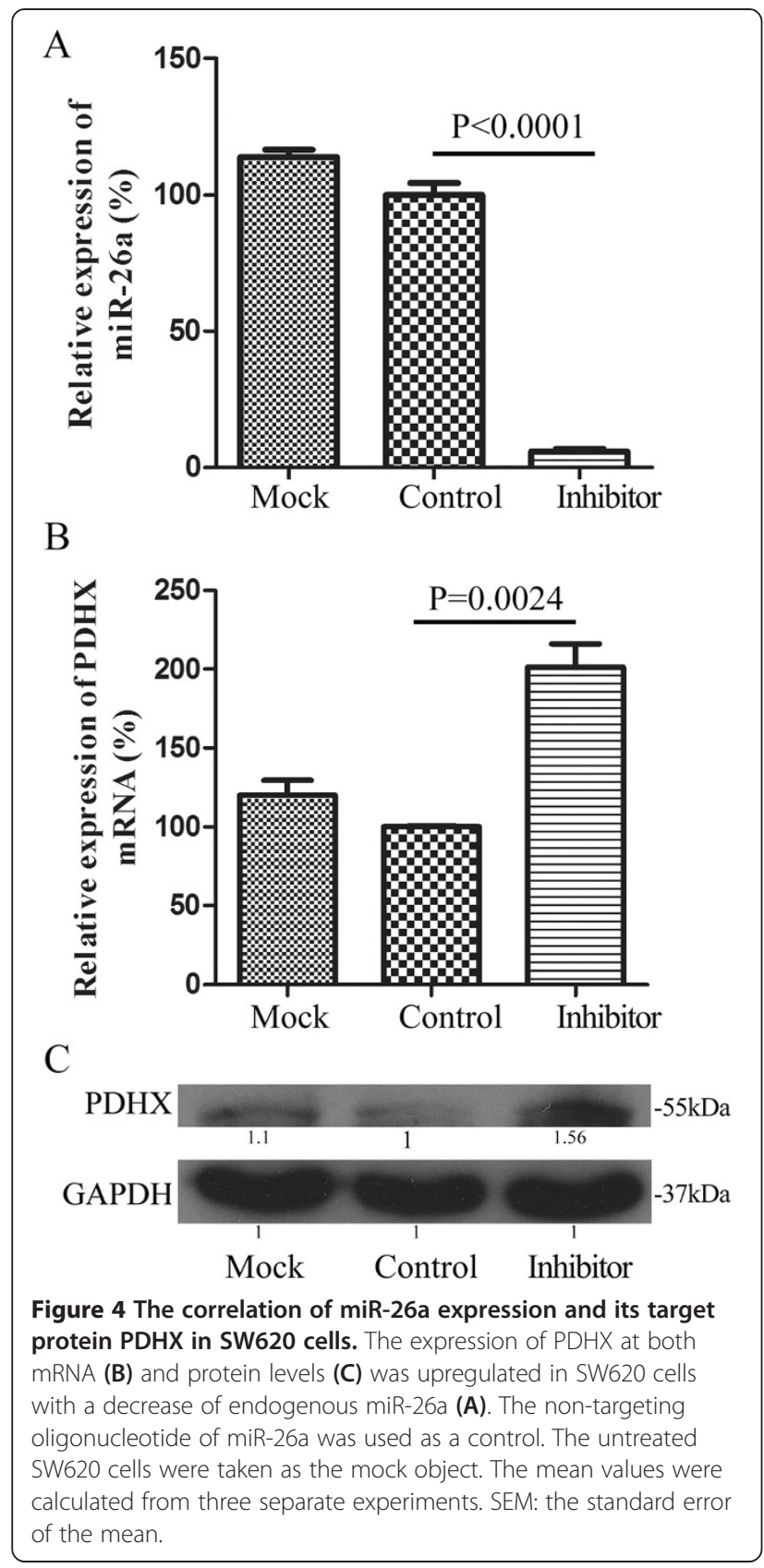

plasmid pmt-PDHX, when the wild type plasmid pwtPDHX was co-transfected with the miR-26a-expressing plasmid pENTR-miR-26a, the ectopically expressing miR-26a bound to the 3' UTR of the wild type plasmid pwt PDHX, then the fused luciferase and the 3'UTR of PDHX was cleaved and subsequently degraded, which resulted in an effective decrease of luciferase activity (Figure 1B). By contrast, in the case of the co-transfection of the mutant plasmid pmt-PDHX with the pENTR-miR26a, the ectopically expressing miR-26a couldn't bind to the 3' UTR of the mutant type plasmid pmt-PDHX, furthermore the luciferase activity did not be decreased.
These data showed that miR-26a modulates the PDHX expression by direct targeting the 3'UTR mRNA of PDHX.

\section{MiR-26a affects cell glucose metabolism}

The glucose consumption and lactate production were gradually increased among the NCM460, HCT116, SW480 and SW620 cells under physiological conditions (Figure 5). Compared with the normal cell line NCM460, each CRC cell line showed a high level of glucose consumption. The glucose uptake was 30.94, 37.38, 44.42 and $110.99 \mu \mathrm{mol}$ respectively for NCM460, HCT116, SW480 and SW620 cells. Moreover, there is a positive correlation between the lactate production and the malignant potential of CRC cells. The lactate production was $38.78 \mu \mathrm{mol}, 70.24 \mu \mathrm{mol}$, $75.63 \mu \mathrm{mol}$ and $195.10 \mu \mathrm{mol}$ from NCM460, HCT116, SW480 to SW620 cells. The lactate content in NCM460 cells was much lower than each of CRC cells. Especially for SW620 cells, it showed more than 5-fold of lactate production than NCM460 cells. In combination with the miR-26a endogenous level in these cells (Figure 2C), it is concluded

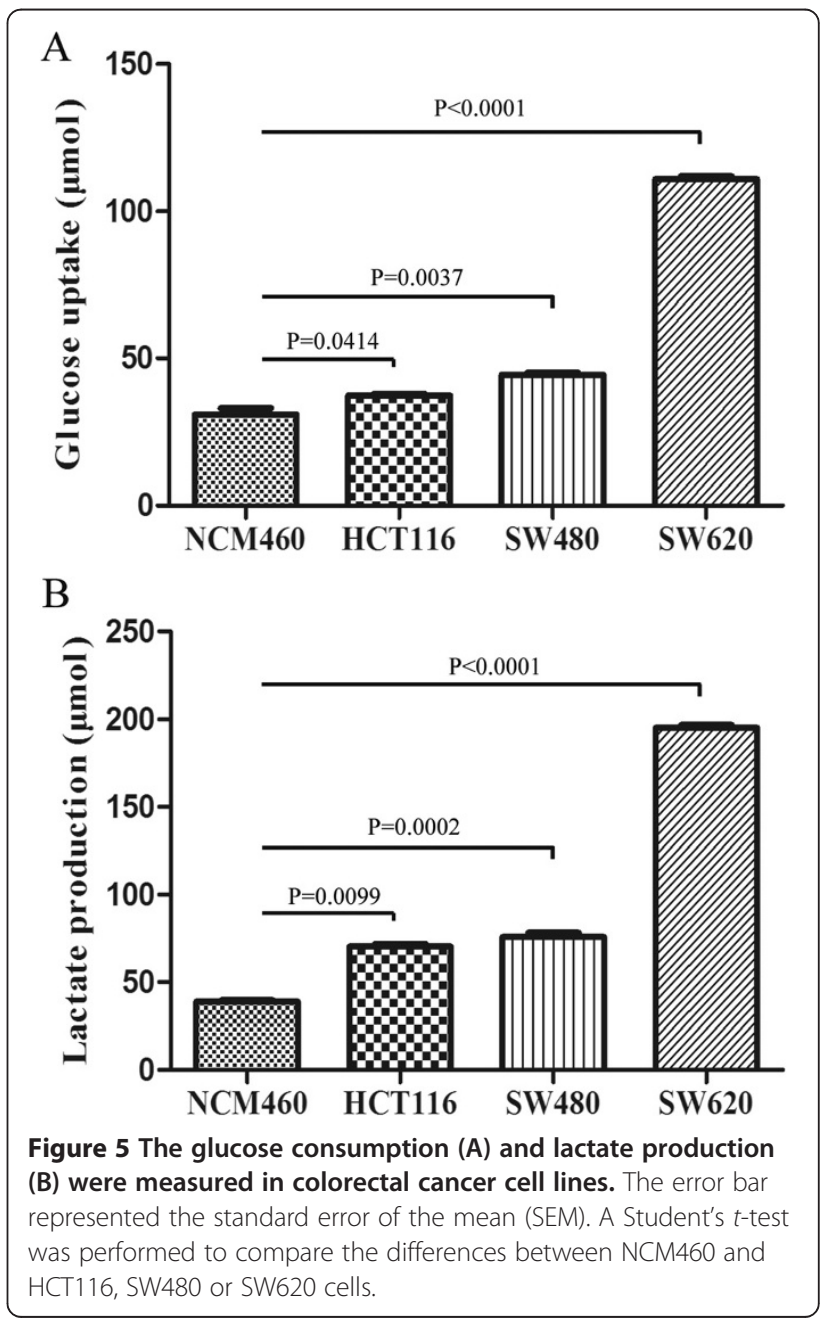


that the overexpression of miR-26a in CRC cells may cause a preference to glycolysis.

In order to investigate miR-26a effects in glucose usage for colon cancer cells, the glucose uptake in HCT116 cells was compared either under the condition of miR-26a overexpression or inhibition. The glucose consumption was increased from $28.86 \mu \mathrm{mol}$ to $53.58 \mu \mathrm{mol}$ in miR-26aoverexpressing HCT116 cells by transfecting with pENTRmiR-26a (Figure 6A), showing an almost 2-fold elevation $(\mathrm{p}<0.05)$. Furthermore, we performed a loss-of-function analysis of miR-26a in glucose consumption by using its inhibitor to silence endogenous miR-26a expression. With loss of miR-26a expression, the glucose usage was decreased from $18.82 \mu \mathrm{mol}$ to $11.25 \mu \mathrm{mol}$ after being treated with miR-26a inhibitor compared to the control (Figure 6B), indicating a 0.59-fold change $(\mathrm{p}<0.005)$. These evidences strongly demonstrated that the expression level of miR-26a is positively associated with the glucose utilization in colon cancer cells. The overexpression of miR-26a enhanced glucose usage. On the contrary, the inhibition of miR-26a expression would reduce glucose consumption.

Considering the direct target molecule of miR-26a, we further detected whether the effects of miR-26a on glucose metabolism were mediated its target gene PDHX. PDHX, also known as E3 binding protein (E3BP), is a non-catalytic subunit of PDH complex $[18,24]$ to catalyze the conversion of pyruvate to acetyl-CoA, thereby linking glycolysis to the citric acid cycle [25]. Since PDHX plays a key role in the conversion of pyruvate to acetyl$\mathrm{CoA}$, we further detected the concentration changes of pyruvate and acetyl-CoA in miR-26a-overexpressing HCT116 cells. As expected, a 1.2-fold increase of pyruvate accumulation (from $8.90 \mu \mathrm{mol}$ to $10.55 \mu \mathrm{mol}$ ) was detected with the ectopic overexpression of miR-26a (Figure 6C), while the amount of acetyl-CoA (from $474.87 \mu \mathrm{mol}$ to $410.26 \mu \mathrm{mol}$ ) in HCT116 cells showed a $14 \%$ decrease (Figure 6E) $(\mathrm{p}<0.05)$. Besides, the expression inhibition of endogenous miR-26a could induce the decrease of pyruvate content. In detail, the pyruvate content in HCT116 cells was decreased from $1.83 \mu \mathrm{mol}$ to $0.66 \mu \mathrm{mol}$, reducing about $63 \%(\mathrm{p}<0.05)$ after being treated with miR-26a inhibitor (Figure 6D). While in the same conditions, the production of acetyl-CoA was effectively increased from $464.87 \mu \mathrm{mol}$ to $554.90 \mu \mathrm{mol}$, raising to almost 1.2 fold $(\mathrm{p}<0.05)$ (Figure 6F).

Similarly, we had also performed the loss-of-function studies in SW620 cells to measure the glucose utilization, pyruvate content and acetyl-CoA production. As shown in Figure 7, when the endogenous miR-26a was inhibited, the glucose uptake was decreased from $47.66 \mu \mathrm{mol}$ to $39.50 \mu \mathrm{mol}$ and the pyruvate content was decreased from $14.36 \mu \mathrm{mol}$ to $2.56 \mu \mathrm{mol}$, with about $20 \%(\mathrm{p}<0.05)$ and $75 \%(\mathrm{p}<0.01)$ reduction respectively. While the acetyl-CoA
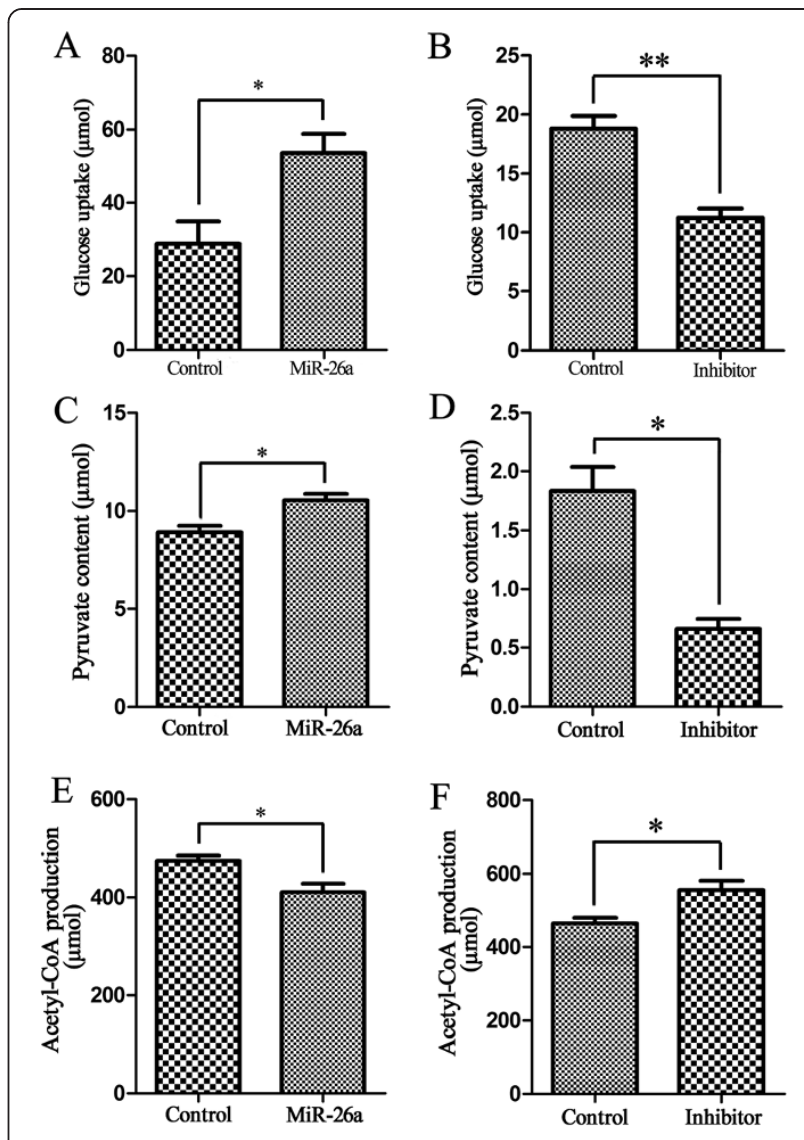

Figure 6 The miR-26a regulates glucose metabolism in HCT116 cells. (A) Overexpression of miR-26a, by transfection of pENTR-miR-26a in HCT116 cells, elevated glucose uptake. The empty vector pENTR-MIRNA was used as the control. (B) The suppressed expression of miR-26a with its inhibitor treatment greatly induced the decrease of glucose uptake. A non-targeting oligonucleotide was used as the control. The miR-26a inhibits the conversion of pyruvate to acetyl-CoA in HCT116 cells.

(C, D) The pyruvate content was increased when HCT116 cells transfected with miR-26a-expressing plasmid pENTR-miR-26a, while the acetly-CoA production was decreased. The empty vector pENTR-MIRNA was used as the control. (E, F) The suppressed expression of miR-26a with its inhibitor treatment induced the decrease of pyruvate content and the up-regulation of the acetly-CoA production. A non-targeting oligonucleotide was used as the control. The average values \pm the standard error of the mean (SEM) of three separate experiments were plotted. ${ }^{*} \mathrm{p}<0.05,{ }^{* *} \mathrm{p}<0.005$.

conversion was increased from $200.28 \mu \mathrm{mol}$ to $252.46 \mu \mathrm{mol}$, with almost a 1.3-fold upregulation $(\mathrm{p}<0.05)$. It indicated that the inhibition of miR-26a in SW620 cells could cause similar glucose metabolic effects that were obtained in HCT116 cells.

All these results have demonstrated that miR-26a inhibits the conversion of pyruvate to acetyl-CoA though directly targeting the PDHX (Figure 8), which accelerates the glucose consumption to undergo aerobic glycolysis for meeting their increased energy and biosynthesis in colon cancer cells. 

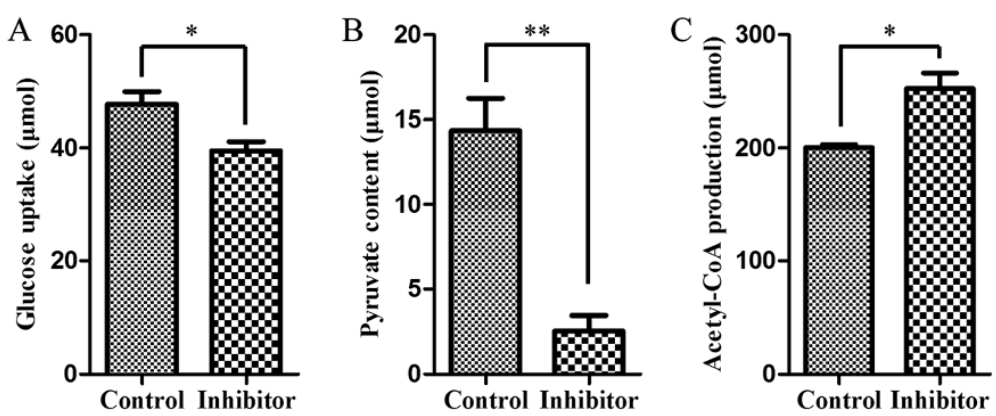

Figure 7 The miR-26a regulates glucose metabolism in SW620 cells. (A) The suppressed expression of miR-26a with its inhibitor treatment greatly induced the decrease of glucose uptake. A non-targeting oligonucleotide was used as the control. The miR-26a inhibits the conversion of pyruvate to acetyl-CoA in SW620 cells. (B, C) The suppressed expression of miR-26a with its inhibitor treatment induced the decrease of pyruvate content and the up-regulation of the acetly-CoA production. A non-targeting oligonucleotide was used as the control. The average values \pm the standard error of the mean (SEM) of three separate experiments were plotted. ${ }^{*} p<0.05,{ }^{* *} p<0.005$.

\section{Discussion}

The role of miR-26a in carcinogenesis appears to be a complicated one, in the sense that both oncogenic and tumor suppressive effects were reported in cancers. For example, miR-26a enhances lung cancer cell metastasis potential via activating AKT pathway by phosphatase and tensin homolog (PTEN) suppression [26]. Other studies find that miR-26a suppresses cell growth and metastasis through IL-6-Stat3 signaling in hepatocellular carcinoma [27]. Our study showed that miR-26a has an increased level in CRC cells, especially it has much higher expression in high-metastasis SW620 cells (Figure 2C). The

\section{Glucose}

\section{Glycolysis}
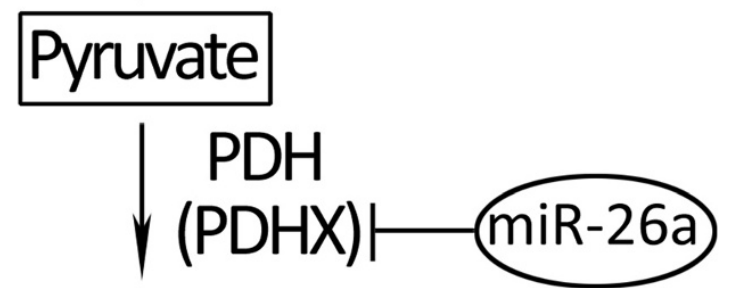

Acetyl-CoA

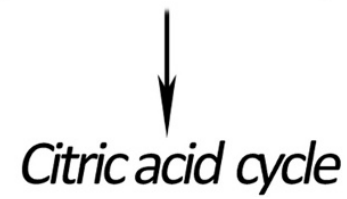

Figure 8 The molecular mechanism of miR-26a involved in glucose metabolism in CRC cells. MiR-26a directly targets the PDHX to suppress the conversion of pyruvate to acetyl-CoA, thus blocking the key step of glycolysis to the citric acid cycle. "f" represents the inhibition effect. finding indicates miR-26a has an oncogenic role in the carcinogenesis of CRC.

More importantly, we also discovered the molecular mechanism of miR-26a involved in glucose metabolism of colon cancer cells. The miR-26a inhibits the expression of PDHX by direct targeting the conserved miR-26a recognition motif of the 3'UTR of PDHX mRNA, which efficiently decreases the process of pyruvate-acetyl-CoA conversion and thus blocks the key step of glycolysis to the citric acid cycle in glucose metabolism. The PDHX is a non-catalytic subunit of the PDH complex, which is located in the mitochondrial matrix and is central to mitochondrial fuel metabolism [28]. The PDH complex catalyzes the irreversible oxidation of pyruvate to acetyl CoA, a rate-limiting step under aerobic conditions for the oxidative removal of glucose and pyruvate and for other 3-carbon metabolites (alanine and lactate) in equilibrium with pyruvate [29]. Except to the non-catalytic subunit PDHX, the PDH complex contains three catalytic subunits (pyruvate dehydrogenase E1, dihydrolipoamide transacetylase E2 and dihydrolipoamide dehydrogenase E3) and two regulatory subunits (E1 kinase and E1 phosphatase). It is known that PDHX is required for tethering E3 dimers to the E2 core and this specific binding is essential for a functional PDH complex. Our results provided experimental evidences to verify the bioinformatics speculation that miR-26a has a high possibility to regulate PDHX in lung cancer [3]. So far, it is the first report to explore the miR-26a-regulated glucose metabolism via targeting PDHX in CRC, which is helpful for understanding miRNA functions in the biochemical processes of the Warburg effect in tumors.

Glucose utilization was changed significantly in HCT116 cells when miR-26a was ectopically overexpressed or inhibited. It seems that miR-26a might prompt glucose uptake to meet the need of energy and biosynthesis in cancer cells. Most mammalian cells import glucose by a process of facilitative diffusion mediated by members of GLUTs family [30]. The effects of miR-26a on the expression and/or the 
translocation of the GLUT proteins could be very interesting to further investigate in our following research. Furthermore, growing evidence has shown that cancer cell metabolism is also controlled by external responses to the tumour microenvironment [9]. For example, cancerassociated fibroblasts have been reported to undergo Warburg metabolism and mitochondrial oxidative stress [31]. They are possibly correlated with metabolic reprogramming of cancer cells toward Warburg metabolism by rapidly expressing carbonic anhydrase IX, which could result in extracellular acidification [32]. These factors will be considered in our further study.

Recently, microRNA molecules are already entering the clinic as potential diagnostic and prognostic biomarkers, as well as therapeutic targets or agents [33]. The miRNAs as important regulators of metabolism have garnered much interest not only from a scientific point of view but also from a clinical perspective [1]. The function of miRNAs on cellular metabolism reveals molecular strategies for controlling metabolic flux by miRNAs in living organisms, thus lighting up one aspect of miRNA therapeutics. The use of miRNAs, such as oligonucleotide complementary [34] or antisense oligonucleotides [35] in miRNA inhibition, to suppress cell metabolism altering will hopefully lead to a new therapeutic strategy for malignant cancer [36,37]. A high rate of glycolysis to obtain energy is a commonly observed feature in cancers, and previous studies usually focus on miRNA roles in targeting the key enzymes of glycolysis, including hexokinase-2 [3], and pyruvate dehydrogenase kinase $[38,39]$. Our data indicated that miR-26a also regulates the process of pyruvate-acetylCoA conversion which is the former reaction of entering the citric acid cycle. Previous studies have shown that miR-26a is significantly associated with disease progression and therapy response in CRC [39-42]. Therefore, the miR-26a is a potential cancer therapeutic target by regulating glucose metabolism in $\mathrm{CRC}$, which also provides a promising anti-neoplasia strategy.

\section{Conclusions}

The expression levels of miR-26a are associated with the malignant degrees of CRC cells. MiR-26a has key roles in regulating glucose metabolism by direct targeting PDHX in CRC cells, which inhibits the conversion of pyruvate to acetyl $\mathrm{CoA}$ in tricarboxylic acid cycle. These novel findings are helpful for understanding the CRC development and indicate a promising anti-neoplasia strategy.

\section{Additional files}

Additional file 1: Table S1. The nucleotide sequences cloned in pENTR-miR-26a.

Additional file 2: Table S2. The full length nucleotide sequences of PDHX 3'UTR cloned in the plasmid pwt-PDHX and pmt-PDHX.

\section{Abbreviations}

miR-26a: microRNA-26a; GLUT: Glucose transporter; UTR: Untranslated region; PDHX: Pyruvate dehydrogenase protein X component; miRNAs: microRNAs; CRC: Colorectal cancer; acetyl-CoA: acetyl coenzyme A.

\section{Competing interests}

The authors declare that they have no competing interests.

\section{Authors' contributions}

Chen B performed most of the experiments and wrote the paper; Liu J performed bioinformatics analysis; Jin X, Liu Y and LU W Co-worked with Xia Z and Yuan Q to perform cellular experiments. Zhao $X$ and Xu N gave suggestions on this project. Liang $S$ conceived, instructed all experiments and revised the paper. All authors read and approved the final manuscript.

\section{Acknowledgements}

This work was financially supported by the grants from National Key Basic Research Program of China (2011CB910703, 2013CB911303), the National 863 High Tech Foundation (2014AA020608), National Natural Sciences Foundation of China (30970654, 31071235), grants for New Century Excellent Talents in University (NCET-10-0595) and specialized research fund for the Doctoral Program of Higher Education (20120181110025). The authors gratefully acknowledged the support of Sichuan Province Program (2010JQ0016, 2012SZ0002)

\section{Author details}

${ }^{1}$ State Key Laboratory of Biotherapy/Collaborative Innovation Center for Biotherapy, West China Hospital, Sichuan University, No.17, the third section of Renmin South Road, Chengdu 610041, P. R. China. ${ }^{2}$ Department of Pharmacology, Medical College, Wuhan University of Science and Technology, Wuhan 430065, P. R. China. ${ }^{3}$ Gynecologic and Pediatric Diseases and Birth Defects of Ministry of Education, West China Second Hospital, Sichuan University, Chengdu 610041, P. R. China. ${ }^{4}$ Laboratory of Cell and Molecular Biology, Cancer Institute and Cancer Hospital, Chinese Academy of Sciences, Beijing 100034, P. R. China.

Received: 16 April 2014 Accepted: 10 June 2014

Published: 16 June 2014

\section{References}

1. Chen B, Li H, Zeng X, Yang P, Liu X, Zhao X, Liang S: Roles of microRNA on cancer cell metabolism. J Transl Med 2012, 10(1):228

2. Pucci S, Mazzarelli P: MicroRNA dysregulation in colon cancer microenvironment interactions: the importance of small things in metastases. Cancer Microenviron 2011, 4(2):155-162.

3. Fang $R$, Xiao T, Fang Z, Sun Y, Li F, Gao Y, Feng Y, Li L, Wang Y, Liu X, Chen H, Liu X, Ji H: MicroRNA-143 (miR-143) regulates cancer glycolysis via targeting hexokinase 2 gene. J Biol Chem 2012, 287(27):23227-23235.

4. Singh PK, Brand RE, Mehla K: MicroRNAs in pancreatic cancer metabolism. Nat Rev Gastroenterol Hepatol 2012, 9(6):334-344.

5. Fei X, Qi M, Wu B, Song Y, Wang Y, Li T: MicroRNA-195-5p suppresses glucose uptake and proliferation of human bladder cancer T24 cells by regulating GLUT3 expression. FEBS Lett 2012, 586(4):392-397.

6. Peschiaroli A, Giacobbe A, Formosa A, Markert EK, Bongiorno-Borbone L, Levine AJ, Candi E, D'Alessandro A, Zolla L, Finazzi Agro A, Melino G: miR-143 regulates hexokinase 2 expression in cancer cells. Oncogene 2013, 32(6):797-802

7. Jordan SD, Krüger M, Willmes DM, Redemann N, Wunderlich FT, Brönneke HS, Merkwirth C, Kashkar H, Olkkonen VM, Böttger T, Braun T, Seibler J, Brüning JC: Obesity-induced overexpression of miRNA-143 inhibits insulin-stimulated AKT activation and impairs glucose metabolism. Nat Cell Biol 2011, 13(4):434-446.

8. Hanahan D, Weinberg RA: Hallmarks of cancer: the next generation. Cell 2011, 144(5):646-674.

9. Cairns RA, Harris IS, Mak TW: Regulation of cancer cell metabolism. Nat Rev Cancer 2011, 11(2):85-95.

10. Volinia S, Calin GA, Liu CG, Ambs S, Cimmino A, Petrocca F, Visone R, Iorio M, Roldo C, Ferracin M, Prueitt RL, Yanaihara N, Lanza G, Scarpa A, Vecchione A, Negrini M, Harris CC, Croce CM: A microRNA expression signature of human solid tumors defines cancer gene targets. Proc Natl Acad Sci U S A 2006, 103(7):2257-2261. 
11. Kulshreshtha R, Ferracin M, Wojcik SE, Garzon R, Alder H, Agosto-Perez FJ, Davuluri R, Liu CG, Croce CM, Negrini M, Calin GA, Ivan M: A microRNA signature of hypoxia. Mol Cell Biol 2007, 27(5):1859-1867.

12. Zhang B, Liu XX, He JR, Zhou CX, Guo M, He M, Li MF, Chen GQ, Zhao Q: Pathologically decreased miR-26a antagonizes apoptosis and facilitates carcinogenesis by targeting MTDH and EZH2 in breast cancer. Carcinogenesis 2011, 32(1):2-9.

13. Chen L, Zheng J, Zhang Y, Yang L, Wang J, Ni J, Cui D, Yu C, Cai Z: Tumorspecific expression of microRNA-26a suppresses human hepatocellular carcinoma growth via cyclin-dependent and -independent pathways. Mol Ther 2011, 19(8):1521-1528.

14. Leeper NJ, Raiesdana A, Kojima Y, Chun HJ, Azuma J, Maegdefessel L, Kundu RK, Quertermous T, Tsao PS, Spin JM: MicroRNA-26a is a novel regulator of vascular smooth muscle cell function. J Cell Physiol 2011, 226(4):1035-1043.

15. Liu B, Wu X, Wang C, Liu Y, Zhou Q, Xu K: MiR-26a enhances metastasis potential of lung cancer cells via AKT pathway by targeting PTEN. Biochim Biophys Acta 2012, 1822(11):1692-1704.

16. Altenberg B, Greulich KO: Genes of glycolysis are ubiquitously overexpressed in 24 cancer classes. Genomics 2004, 84(6):1014-1020.

17. Alcarraz-Vizan G, Sanchez-Tena S, Moyer MP, Cascante M: Validation of NCM460 cell model as control in antitumor strategies targeting colon adenocarcinoma metabolic reprogramming: Trichostatin $\mathrm{A}$ as a case study. Biochim Biophys Acta 2013, 1840(6):1634-1639.

18. Krek A, Grun D, Poy MN, Wolf R, Rosenberg L, Epstein EJ, MacMenamin P, da Piedade I, Gunsalus KC, Stoffel M, Rajewsky N: Combinatorial microRNA target predictions. Nat Genet 2005, 37(5):495-500.

19. Jiang S, Zhang LF, Zhang HW, Hu S, Lu MH, Liang S, Li B, Li Y, Li D, Wang ED, Liu MF: A novel miR-155/miR-143 cascade controls glycolysis by regulating hexokinase 2 in breast cancer cells. EMBO J 2012, 31(8):1985-1998.

20. Sze CW, Li C: Inactivation of bb0184, which encodes carbon storage regulator $\mathrm{A}$, represses the infectivity of Borrelia burgdorferi. Infect Immun 2011, 79(3):1270-1279.

21. Moyer MP, Manzano LA, Merriman RL, Stauffer JS, Tanzer LR: NCM460, a normal human colon mucosal epithelial cell line. In Vitro Cell Dev Biol Anim 1996, 32(6):315-317.

22. Bhattacharyya NP, Skandalis A, Ganesh A, Groden J, Meuth M: Mutator phenotypes in human colorectal carcinoma cell lines. Proc Natl Acad Sci U S A 1994, 91(14):6319-6323.

23. Xue H, Lü B, Zhang J, Wu M, Huang Q, Wu Q, Sheng H, Wu D, Hu J, Lai M: Identification of serum biomarkers for colorectal cancer metastasis using a differential secretome approach. J Proteome Res 2009, 9(1):545-555.

24. Tajir M, Arnoux JB, Boutron A, Elalaoui SC, De Lonlay P, Sefiani A, Brivet M: Pyruvate dehydrogenase deficiency caused by a new mutation of PDHX gene in two Moroccan patients. Eur J Med Genet 2012, 55(10):535-540.

25. Mine M, Brivet M, Schiff M, de Baulny HO, Chuzhanova N, Marsac C: A novel gross deletion caused by non-homologous recombination of the PDHX gene in a patient with pyruvate dehydrogenase deficiency. Mol Genet Metab 2006, 89(1-2):106-110.

26. Huse JT, Brennan C, Hambardzumyan D, Wee B, Pena J, Rouhanifard SH, Sohn-Lee C, le Sage C, Agami R, Tuschl T, Holland EC: The PTEN-regulating microRNA miR-26a is amplified in high-grade glioma and facilitates gliomagenesis in vivo. Genes Dev 2009, 23(11):1327-1337.

27. Yang X, Liang L, Zhang XF, Jia HL, Qin Y, Zhu XC, Gao XM, Qiao P, Zheng Y, Sheng YY, Wei JW, Zhou HJ, Ren N, Ye QH, Dong QZ, Qin LX: MicroRNA26a suppresses tumor growth and metastasis of human hepatocellular carcinoma by targeting interleukin-6-Stat3 pathway. Hepatology 2013, 58(1):158-170.

28. Stacpoole PW: The pyruvate dehydrogenase complex as a therapeutic target for age-related diseases. Aging Cell 2012, 11:371-377.

29. Margineantu DH, Brown RM, Brown GK, Marcus AH, Capaldi RA: Heterogeneous distribution of pyruvate dehydrogenase in the matrix of mitochondria. Mitochondrion 2002, 1(4):327-338.

30. Thorens B, Mueckler M: Glucose transporters in the 21 st century. Am J Physiol Endocrinol Metab 2010, 298:E141-E145.

31. Fiaschi T, Marini A, Giannoni E, Taddei M, Gandellini P, De Donatis A, Lanciotti M, Serni S, Cirri P, Chiarugi P: Reciprocal metabolic reprogramming through lactate shuttle coordinately influences tumor-stroma interplay. Cancer Res 2012, 72:5130-5140.
32. Fiaschi T, Giannoni E, Taddei M, Cirri P, Marini A, Pintus G, Nativi C, Richichi B, Scozzafava A, Carta F, Torre E, Supuran CT, Chiarugi P: Carbonic anhydrase IX from cancer-associated fibroblasts drives epithelial-mesenchymal transition in prostate carcinoma cells. Cell Cycle 2013, 12:1791-1801.

33. Jansson MD, Lund AH: MicroRNA and cancer. Mol Oncol 2012, 6(6):590-610.

34. Lanford RE, Hildebrandt-Eriksen ES, Petri A, Persson R, Lindow M, Munk ME Kauppinen S, Orum H: Therapeutic silencing of microRNA-122 in primates with chronic hepatitis C virus infection. Science 2010, 327(5962):198-201.

35. Stenvang J, Petri A, Lindow M, Obad S, Kauppinen S: Inhibition of microRNA function by antimiR oligonucleotides. Silence 2012, 3(1):1.

36. Rossi JJ: New hope for a microRNA therapy for liver cancer. Cell 2009, 137(6):990-992.

37. Pereira DM, Rodrigues PM, Borralho PM, Rodrigues CM: Delivering the promise of miRNA cancer therapeutics. Drug Discov Today 2013, 18(5-6):282-289.

38. El Ouaamari A, Baroukh N, Martens GA, Lebrun P, Pipeleers D, van Obberghen E: miR-375 targets 3'-phosphoinositide-dependent protein kinase-1 and regulates glucose-induced biological responses in pancreatic beta-cells. Diabetes 2008, 57(10):2708-2717.

39. Kluza J, Corazao-Rozas P, Touil Y, Jendoubi M, Maire C, Guerreschi P, Jonneaux A, Ballot C, Balayssac S, Valable S, Corroyer-Dulmont A, Bernaudin M, Malet-Martino M, de Lassalle EM, Maboudou P, Formstecher P, Polakowska R, Mortier L, Marchetti P: Inactivation of the HIF-1alpha/PDK3 signaling axis drives melanoma toward mitochondrial oxidative metabolism and potentiates the therapeutic activity of pro-oxidants. Cancer Res 2012 72(19):5035-5047.

40. Iorio MV, Croce CM: MicroRNA dysregulation in cancer: diagnostics, monitoring and therapeutics. A comprehensive review. EMBO Mol Med 2012, 4(3):143-159.

41. Boni V, Zarate R, Villa JC, Bandres E, Gomez MA, Maiello E, Garcia-Foncillas J, Aranda E: Role of primary miRNA polymorphic variants in metastatic colon cancer patients treated with 5-fluorouracil and irinotecan. Pharmacogenomics J 2011, 11(6):429-436.

42. Di Leva G, Briskin D, Croce CM: MicroRNA in cancer: new hopes for antineoplastic chemotherapy. Ups J Med Sci 2012, 117(2):202-216.

doi:10.1186/1471-2407-14-443

Cite this article as: Chen et al:: MicroRNA-26a regulates glucose metabolism by direct targeting PDHX in colorectal cancer cells. BMC Cancer 2014 14:443.

\section{Submit your next manuscript to BioMed Central and take full advantage of:}

- Convenient online submission

- Thorough peer review

- No space constraints or color figure charges

- Immediate publication on acceptance

- Inclusion in PubMed, CAS, Scopus and Google Scholar

- Research which is freely available for redistribution 